Part 1 Cosmic Dawn and Epoch of Reionization: theory and simulations 


\title{
Reionization and Cosmic Dawn: theory and simulations
}

\author{
Andrei Mesinger \\ Scuola Normale Superiore, \\ Piazza dei Cavalieri, 7 \\ 56126 Pisa, Italy \\ email: andrei.mesinger@sns.it
}

\begin{abstract}
We highlight recent progress in the sophistication and diversification of the simulations of cosmic dawn and reionization. The application of these modeling tools to recent observations has allowed us narrow down the timing of reionization. The midpoint of reionization is constrained to $z=7.6_{-0.7}^{+0.8}(1 \sigma)$, with the strongest constraints coming from the optical depth to the CMB measured with the Planck satellite and the first detection of ongoing reionization from the spectra of the $z=7.1$ QSOs ULASJ1120+0641. However, we still know virtually nothing about the astrophysical sources during the first billion years. The revolution in our understanding will be led by upcoming interferometric observations of the cosmic $21-\mathrm{cm}$ signal. The properties of the sources and sinks of UV and X-ray photons are encoded in the $3 \mathrm{D}$ patterns of the signal. The development of Bayesian parameter recovery techniques, which tap into the wealth of the $21-\mathrm{cm}$ signal, will soon usher in an era of precision astrophysical cosmology.
\end{abstract}

Keywords. cosmology: theory, early universe, diffuse radiation, large-scale structure of universe

\section{Introduction}

The first billion years of our Universe witnessed the birth of the first stars and galaxies. These galactic ancestors were likely much smaller than present-day galaxies, yet they could have hosted stars and stellar black holes far more massive than typically found today. The light emerging from these galaxies started spreading through our dark and cold Universe, heralding the Cosmic Dawn (CD). This radiation influenced other nascent galaxies, as well as heated and ionized the pervasive intergalactic medium (IGM). This process culminated in the final major phase change of our Universe, the Epoch of Reionization (EoR), with more than $99.99 \%$ of the atoms in the IGM becoming ionized. The $\mathrm{CD}$ and EoR contain the answers to some fundamental questions: When did the first generations of galaxies form? What were their properties? How did they interact with each other? What is the structure of the IGM? What is the thermal and ionization history of the baryons?

Answering these questions is very challenging. Most of the first galaxies are likely far too faint to be directly observed with upcoming instruments, and their properties must be inferred indirectly. Interpreting the current scant observations remains controversial. The problem is two-fold. Firstly, we are faced with a huge range of relevant scales (i.e. dynamic range). Ultimately small-scale physics governing the birth and death of stars is responsible for driving large-scale radiation fields. The EoR/CD is inhomogeneous on scales of hundreds of $M p c$; correctly interpreting current and upcoming observations requires us to capture these inhomogeneities. Secondly, we know very little about galaxy formation in the early Universe. The complexities of the relevant physics combined with 

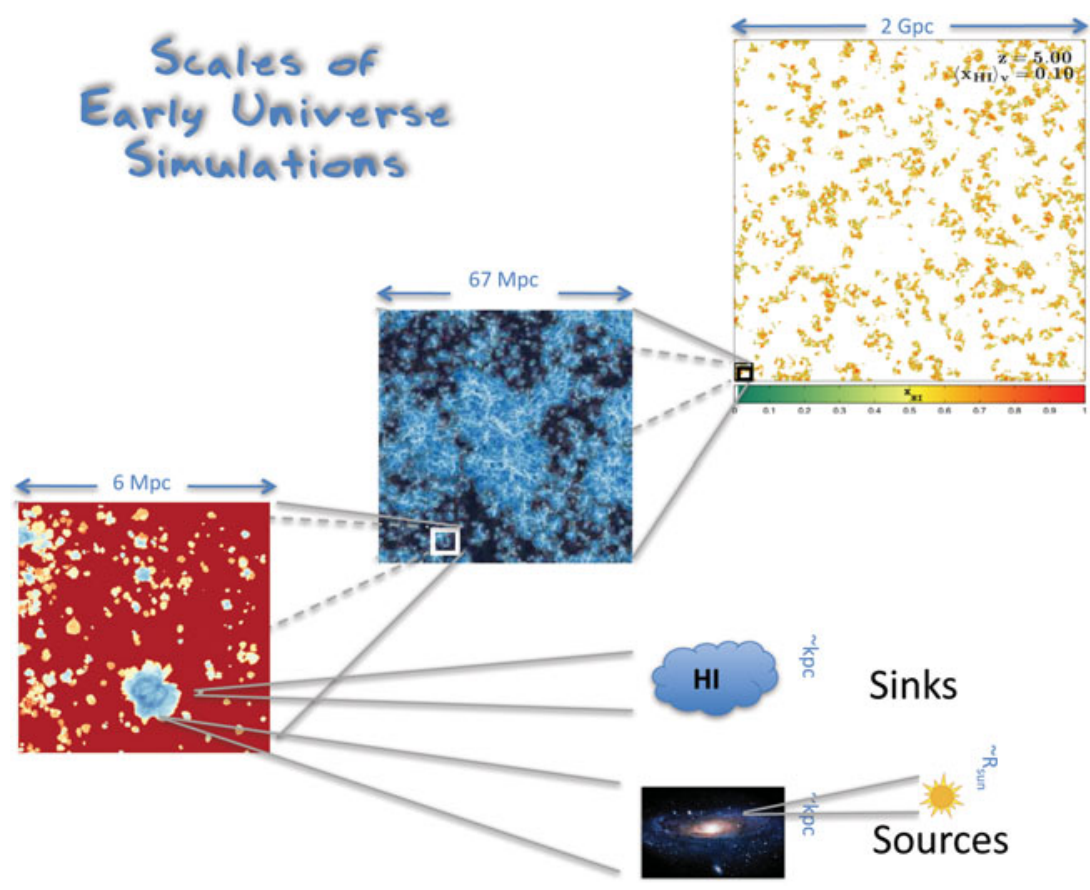

Figure 1. Illustration of the various scales and corresponding cosmological simulation tools. Stars and gas clumps ultimately drive radiation fields which are inhomogeneous on cosmological scales (hundreds of Mpc). From bottom left to top right, we show slices through ionization fields computed with hydrodynamic radiative transfer simulations (Xu et al., 2016), N-body with post-processed radiative transfer (Dixon et al., 2016), and semi-numerical simulations (Mesinger, 2010). Note that the simulations are independent; zoom-ins are only to give a sense of relative scale.

the lack of detailed observations leaves us with an enormous parameter space of astrophysical uncertainties.

As a result, recent years have seen a shift towards diversification of cosmological simulations of the EoR/CD. These can sacrifice resolution and physical complexity for speed and scale, allowing us to adapt to the exigencies of each particular observation ( $(2)$. This diverse simulation toolkit allowed us to robustly interpret the latest observations of the cosmic microwave background (CMB) as well as high- $z$ Lyman alpha emitting galaxies and quasars. Consensus is emerging on the timing of reionization ( $\$ 3$ ). However, we still know almost nothing about the details of this process and the galaxies and/or AGN thought to be driving it. Luckily, the next few years will see a revolution in CD/EoR studies, enabled by the interferometry of the $21-\mathrm{cm}$ line of neutral hydrogen $(\S 4)$.

\section{The toolkit for cosmological simulations}

The enormous computational requirements of the EoR/CD has led to a diversification of cosmological simulation tools, as mentioned above and illustrated in Fig. 1 (see also the review in Trac and Gnedin, 2011). These can vary in the treatment of both: (i) the source/sink fields (e.g. galaxies and recombining clumps), and (ii) the radiate transfer (RT).

The most realistic treatment of (i) uses coupled N-body and hydrodynamic codes, while the most realistic treatment of (ii) uses ray-tracing approaches. Such simulations are usually limited to scales of $\sim 1-10 \mathrm{Mpc}$, if they wish to resolve the bulk of the early 
galaxy population (e.g. Xu et al., 2016). These physics-rich simulations are invaluable in resolving the very first, molecular-cooled galaxies (residing in halos with masses of $\sim 10^{6}-10^{8} M_{\odot}$ ), as well as studying processes such as radiative feedback, metal pollution, stochastic star formation and recombining clumps in the IGM. However, cosmological simulations still cannot resolve the sub-structure of stellar environments, turbulence and the early phases of SNe explosions, which means that results still depend on sub-grid prescriptions, albeit with fewer and more physically motivated "tuning knobs" than those approaches discussed below. Moreover, these simulations are difficult to calibrate, as they usually cannot be run to moderate redshifts nor do they capture the majority of the galaxies we can observe.

More approximate treatments of either (i) or (ii) can extend EoR/CD simulations to $\sim 100 \mathrm{Mpc}$ scales (e.g. Trac and Cen, 2007, Iliev et al., 2014, Baek et al., 2010, Ocvirk et al., 2016, Kakiichi et al., 2017). These begin to approach the scales needed to statistically sample the expected distribution of ionized regions during the EoR. This is achieved generally by not resolving the ISM of galaxies and/or removing hydrodynamics completely. As a result, galaxy properties such as the star formation rates and ionizing luminosities must be assigned to DM halos as input, reducing their predictive ability. Nevertheless, given several of these input prescriptions, these simulations can be used to distinguish between the resulting ionization fields and associated observables. Monte Carlo based RT methods allow us to achieve large scales while keeping medium resolution hydrodynamics, by reducing either the source population to only rare host dark matter or the number of emitted photon packets per galaxy (e.g. Baek et al., 2010, Partl et al., 2011). Moment based RT methods (e.g. the Optically Thin Variable Eddington Tensor approach of Gnedin and Abel, 2001) achieve comparable scales with more sources, but at the cost of increased diffusion of the radiation fields. However most RT approaches show reasonable agreement (Iliev et al., 2006).

Recent years have witnessed the advent of even more approximate, so-called "seminumerical" schemes (e.g. Zahn et al., 2007, Mesinger and Furlanetto, 2007, Geil and Wyithe, 2008, Choudhury et al., 2009). These replace RT of ionizing photons with an excursion-set approach (Furlanetto et al., 2004a), in which the cumulative number of ionizing photons produced are compared against the number of neutral atoms in regions of decreasing spherical scales. Photons with longer mean free paths, like X-rays and soft UV, can be tracked by integrating back along the light-cone (Santos et al., 2010, Mesinger et al., 2011, Fialkov et al., 2013). Many of these schemes replace even $N$ body codes, computing the source fields directly from the density field with excursionset halo mass functions (Bond et al., 1991, Lacey and Cole, 1993). This enables virtually unlimited dynamic range, allowing EoR/CD simulations to approximately match the field of view of upcoming 21-cm interferometers (Mesinger et al., 2016). Because of their speed semi-numerical codes enable rapid exploration of the large parameter space of astrophysical uncertainties. However, the radiation fields become inaccurate on small scales (§Mpc; Zahn et al., 2011), and as with all simulations which do not resolve galactic star-formation, the inputted source properties are parametric.

Finally, "hybrid" techniques have even been developed, which combine some of the advantages and disadvantages of the above. For example, the large-scale patchiness of reionization captured by semi-numerical codes can be combined (in a statistical fashion) with small-scale IGM structure predicted by hydrodynamic simulations (Mesinger et al., 2015, Choudhury et al., 2015). This approach has been used to study the impact of IGM damping wing absorption to Lyman alpha emitting galaxies, which can be sensitive to neutral hydrogen structure over a wide range of scales. As another example, Mutch et al., 2016 incorporate a semi-analytic star-formation model, based on $N$-body 
merger trees, within a semi-numeric treatment of reionization, allowing for a more physical parametrization of source properties.

The diversification of these tools was driven by the challenge of finding the right tool for the job. There is no "one size fits all" for EoR simulations. Each analysis should be tailored to a given observable, focusing on the most relevant physics and scales (e.g. smallscale IGM structure, Ly $\alpha$ forest, large-scale EoR structure, ISM sub-structure, molecular cooled halos, etc.), and parametrizing the missing physics. In the next section, we will discuss how applying these simulation tools to current observations allowed us to start narrowing down the history of reionization.

\section{The current state of knowledge: timing of reionization}

Our current knowledge about the EoR stems from two classes of probes: (i) integral constraints from the CMB; and (ii) astrophysical "flashlights" which illuminate the intervening IGM. The most important CMB constraint comes in the form of the Thompson scattering optical depth to the last scattering surface, $\tau_{e} \cdot \dagger$ The latest results from the Planck satellite give $\tau_{e}=0.058 \pm 0.012(1 \sigma)$ Planck Collaboration, 2016, implying a later EoR than previous estimates. Since $\tau_{e}$ is an integral constraint, using it to infer the EoR requires the assumption of a functional form for the redshift evolution of the average neutral fraction, $\bar{x}_{\mathrm{HI}}(\mathrm{z})$.

On the other hand, if found at a sufficiently high- $z$, astrophysical flashlights allow us to glimpse the state of the EoR at a given redshift. The "color" of these flashlights is generally Lyman alpha, which is the strongest emission line and is sensitive to the presence of neutral hydrogen. While Lyman alpha absorption in the IGM produces a Ly $\alpha$ forest in the spectra of moderate redshift QSOs, the line saturates quickly saturates going beyond $z \gtrsim 6$ (e.g. Fan et al., 2006. However, during the EoR, the Lorentzian damping wings of the line can provide a sufficiently high optical depth to absorb photons on the red side of the Lyman alpha resonance. The imprint of this EoR damping wing can be studied in individual bright QSO spectra (e.g. Bolton et al., 2011), or in ensembleaveraged properties of fainter galaxy spectra (e.g. Dijkstra, 2014).

In Fig. 2, we summarize the current state of knowledge on the history of reionization (taken from Greig and Mesinger, 2017; see also similar results by Mitra et al., 2015, Price et al., 2016). Fitting a physically-motivated basis set of $\bar{x}_{\mathrm{HI}}(z)$ to current observations, these authors constrain the epochs corresponding to an average neutral fraction of (75, $50,25)$ per cent, to $z=\left(8.52_{-0.87}^{+0.96}, 7.57_{-0.73}^{+0.78}, 6.82_{-0.71}^{+0.78}\right),(1-\sigma)$. The strongest constraints here come from the first detection of ongoing reionization, obtained from the spectra of the $z=7.1$ QSOs ULASJ1120+0641: $\bar{x}_{\mathrm{HI}}(z=7.1)=0.4_{-0.32}^{+0.41}(2-\sigma)$; see also the recent work by Mason et al., 2017 who obtain comparable limits from the disappearance of Lyman alpha emitting galaxies beyond $z \gtrsim 6$ (not shown in the figure).

The past few years have witnessed dramatic progress in our understanding of the timing of reionization. However, virtually nothing is known about the sources (and sinks) which govern this process. The true revolution in our understanding of the first billion years will come with upcoming 21-cm interferometers.

$\dagger$ Alternative probes such as E-mode polarization as a function of angular scale (e.g. Mortonson and $\mathrm{Hu}, 2008$ ), the patchiness of $\tau_{e}$ (e.g. Dvorkin and Smith, 2009), the kinetic Sunyaev-Zel'dovich signal from patchy reionization (e.g. Mesinger et al., 2012), could yield interesting results in the future provided systematics can be controlled (see the review of Reichardt, 2016) 


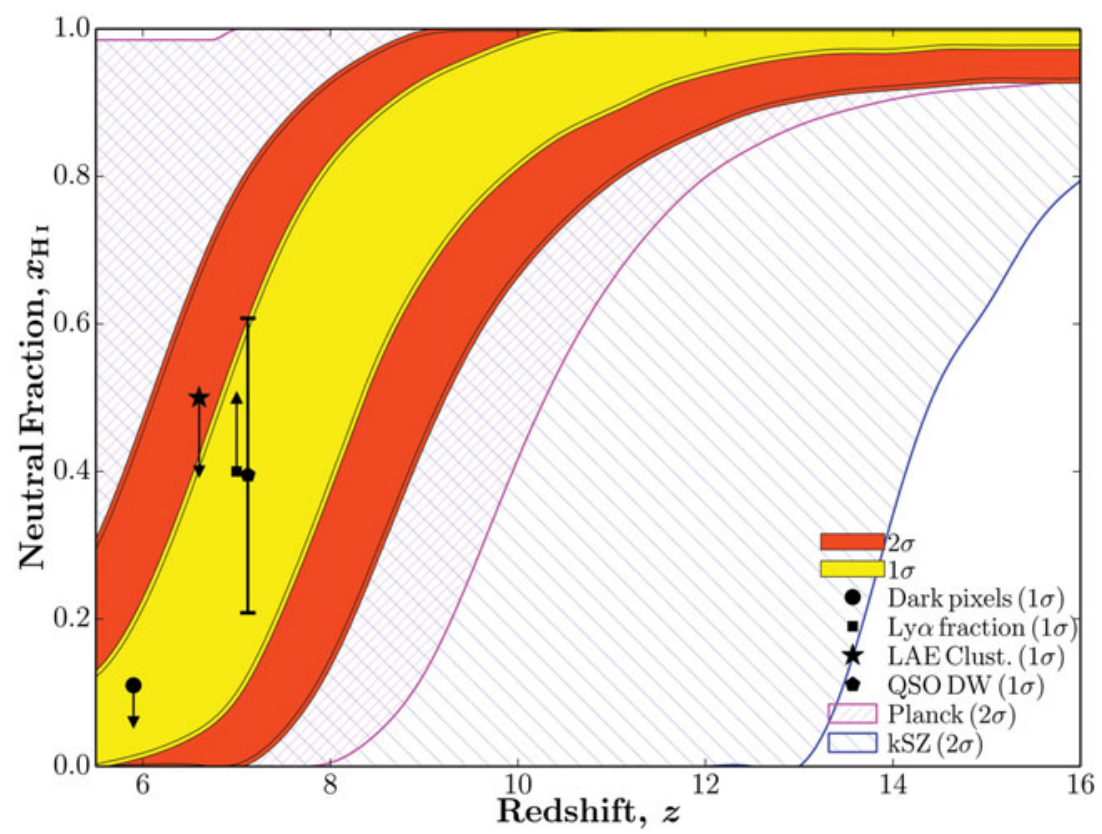

Figure 2. The global history of reionization. A physically-motivated EoR model was sampled, with the likelihood of each resulting $\bar{x}_{\mathrm{HI}}(z)$ curve provided by current observations. The figure is taken from Greig and Mesinger, 2017 (see also similar results by Mitra et al., 2015, Price et al., 2016).

\section{Future potential: the cosmic $21 \mathrm{~cm}$ signal}

The spin flip transition of HI, resulting in the emission of a photon with a wavelength of 21-cm, is an extremely powerful probe of the EoR and CD (see the review of Furlanetto et al., 2006). The cosmic neutral hydrogen can be seen in contrast against the CMB. The 21-cm signal is sensitive to both the ionization and thermal state of the cosmic gas, and it contains both astrophysical and cosmological terms. Since it is a line signal, with a given frequency corresponding to a redshift, upcoming interferometers can provide a 3D map of the first billion years of our Universe!

Current interferometers are already taking data, hoping for a statistical detection of the EoR. These include the Low Frequency Array (LOFAR; van Haarlem et al., 2013), Murchison Wide Field Array (MWA; Tingay et al., 2013), and the Precision Array for Probing the Epoch of Reionization (PAPER; Parsons et al., 2010). Next-generation interferometers, the Hydrogen Epoch of Reionization Arrays (HERA; DeBoer et al., 2016), and the Square Kilometre Array (SKA; e.g. Koopmans et al., 2015) will be coming on-line soon, with sufficient sensitivity to capture even the earlier stages of the CD, and could even provide tomographic maps of the first billion years.

How do we tap into this wealth of information? The patterns of the 21-cm signal (i.e. the fluctuations in the ionization and temperature of the IGM) encode the UV and $\mathrm{X}$-ray properties of the first galaxies! For example, if star-formation is only efficient in relatively massive galaxies, the cosmic HII regions should be relatively large and isolated, at a given stage in the EoR (e.g. Furlanetto et al., 2004b, McQuinn et al., 2007, Iliev et al., 2012). Efficient self-shielding of gas clumps in the IGM can produce an opposite effect, sapping the growth of large HII regions (e.g. McQuinn et al., 2007, Sobacchi and Mesinger, 2014). Similarly, the smoothness of the earlier epoch of heating would allow us 

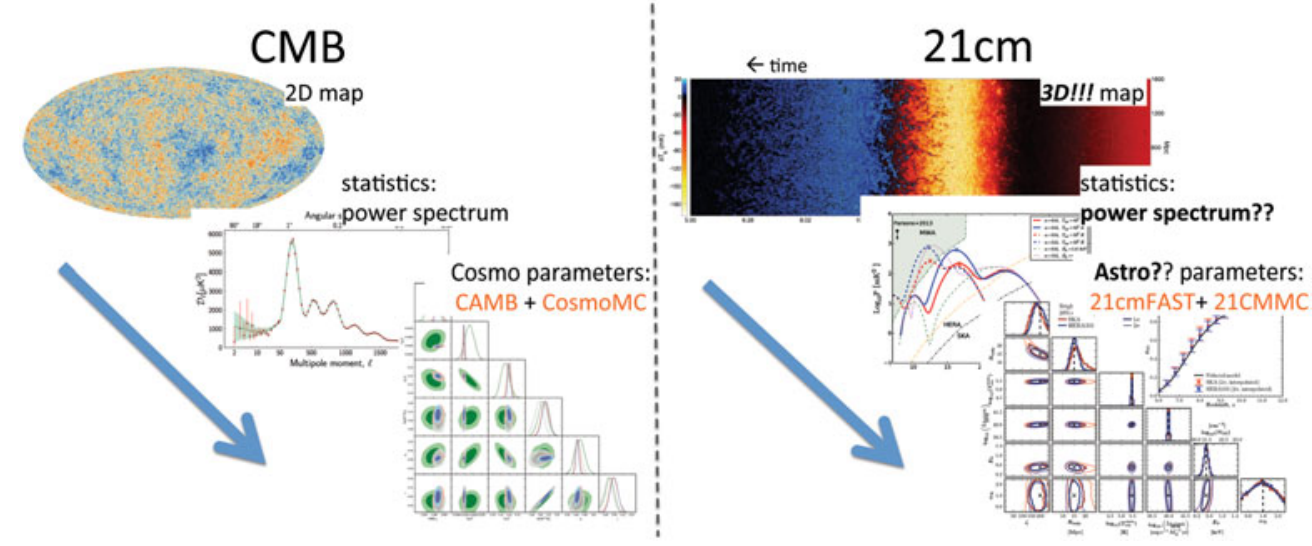

Figure 3. Schematic illustrating the parallels between the established Bayesian parameter recovery with the $\mathrm{CMB}$, and the planed parameter recovery with the cosmic $21 \mathrm{~cm}$ signal.

to discriminate between different high-energy processes in the first galaxies (e.g. Pacucci et al., 2014, Fialkov et al., 2014).

How do we quantify this? Here we can take inspiration from the widely-successful Bayesian framework for cosmological parameter estimation from the CMB (see the left panel of Fig. 3). A $2 \mathrm{D}$ map of the $\mathrm{CMB}$ is compressed into a summary statistic (the angular power spectra). Then an MCMC sampler (e.g. CosmoMC; Lewis and Bridle, 2002) is used to constrain physical cosmological parameters, comparing corresponding theoretical power spectra (with, e.g. CMBFAst/CAMB; Seljak and Zaldarriaga, 1996, Lewis et al., 2000) against the observations.

Astrophysics with the 21-cm signal is similar in many ways (see the right panel of Fig. 3 ). Here we start with a $3 D$ map of the 21 -cm signal, containing many orders of magnitude more modes than the CMB (for a review of the associated observational challenges, see the chapter by S. Zaroubi in this volume). This signal is then compressed into summary statistics. Although the power spectrum is a natural choice for a summary statistic, it is not clear whether this is optimal because the 21-cm signal (unlike the CMB) is highly nonGaussian. Complementary information could be provided by alternate statistics, such as the 1-point distribution (e.g. Barkana and Loeb, 2008, Watkinson and Pritchard, 2014), the bispectrum (e.g. Bharadwaj and Pandey, 2005, Shimabukuro et al., 2016, Majumdar et al., 2017), HII region size characterizations (e.g. Zahn et al., 2007, Mesinger and Furlanetto, 2007, Giri et al., 2018), etc. Finally, an MCMC sampler (e.g. 21CMMC; Greig and Mesinger, 2015) can be used to constrain astrophysical parameters, by comparing corresponding theoretical summary statistic (e.g. using 21CMFAST; Mesinger et al., 2011) against the observations.

\section{Conclusions}

The past few years have witnessed substantial progress in the modeling of the epoch of reionization and the cosmic dawn. Simulations have not only become more sophisticated, but have evolved and diversified to best suit their intended use. We now have many techniques for radiative transfer and how the radiation is coupled to the underlying source and sink fields.

Careful application of these tools to current observations allowed us to get an idea of when reionization occurred (e.g. the midpoint is constrained to $z=7.6_{-0.7}^{+0.8}$ at $1 \sigma$ ). The 
strongest constraints come from the optical depth to the CMB, and the first detection of ongoing reionization from the spectra of the $z=7.1$ QSOs ULASJ1120+0641.

Unfortunately, our knowledge does not extend far beyond the timing. We know virtually nothing about the galaxies, AGN, and gas clumps which are the dominant actors in the EoR and CD. Luckily, upcoming 21-cm interferometers will open-up an astrophysical treasure trove, providing a 3D map of the process. The properties of astrophysical sources and sinks are encoded in the patterns of the 21-cm signal. Bayesian frameworks are being developed which can tap into this physical bounty, providing astrophysical parameter constraints. The era of precision astrophysical cosmology is on our doorsteps!

\section{References}

Baek, S., Semelin, B., Di Matteo, P., Revaz, Y., \& Combes, F. (2010). Reionization by UV or X-ray sources. $A \mathscr{G} A, 523: \mathrm{A} 4$.

Barkana, R. \& Loeb, A. (2008). The difference PDF of 21-cm fluctuations: a powerful statistical tool for probing cosmic reionization. MNRAS, 384:1069-1079.

Bharadwaj, S. \& Pandey, S. K. (2005). Probing non-Gaussian features in the HI distribution at the epoch of re-ionization. MNRAS, 358:968-976.

Bolton, J. S., Haehnelt, M. G., Warren, S. J., Hewett, P. C., Mortlock, D. J., Venemans, B. P., McMahon, R. G., \& Simpson, C. (2011). How neutral is the intergalactic medium surrounding the redshift $\mathrm{z}=7.085$ quasar ULAS J1120+0641? MNRAS, 416:L70-L74.

Bond, J. R., Cole, S., Efstathiou, G., \& Kaiser, N. (1991). Excursion set mass functions for hierarchical Gaussian fluctuations. ApJ, 379:440-460.

Choudhury, T. R., Haehnelt, M. G., \& Regan, J. (2009). Inside-out or outside-in: the topology of reionization in the photon-starved regime suggested by Ly $\alpha$ forest data. MNRAS, 394:960977.

Choudhury, T. R., Puchwein, E., Haehnelt, M. G., \& Bolton, J. S. (2015). Lyman $\alpha$ emitters gone missing: evidence for late reionization? MNRAS, 452:261-277.

DeBoer, D. R. et al. (2016). Hydrogen Epoch of Reionization Array (HERA). ArXiv eprints:1606.07473.

Dijkstra, M. (2014). Lyman Alpha Emitting Galaxies as a Probe of Reionization. ArXiv eprints:1406.7292.

Dixon, K. L., Iliev, I. T., Mellema, G., Ahn, K., \& Shapiro, P. R. (2016). The large-scale observational signatures of low-mass galaxies during reionization. MNRAS, 456:3011-3029.

Dvorkin, C. \& Smith, K. M. (2009). Reconstructing patchy reionization from the cosmic microwave background. PRD, 79(4):043003-+.

Fan, X. et al. (2006). Constraining the Evolution of the Ionizing Background and the Epoch of Reionization with z 6 Quasars. II. A Sample of 19 Quasars. AJ, 132:117-136.

Fialkov, A., Barkana, R., \& Visbal, E. (2014). The observable signature of late heating of the Universe during cosmic reionization. Nature, 506:197-199.

Fialkov, A., Barkana, R., Visbal, E., Tseliakhovich, D., \& Hirata, C. M. (2013). The 21-cm signature of the first stars during the Lyman-Werner feedback era. MNRAS, 432:2909 2916.

Furlanetto, S. R., Hernquist, L., \& Zaldarriaga, M. (2004a). Constraining the topology of reionization through Ly $\alpha$ absorption. MNRAS, 354:695-707.

Furlanetto, S. R., Oh, S. P., \& Briggs, F. H. (2006). Cosmology at low frequencies: The $21 \mathrm{~cm}$ transition and the high-redshift Universe. Physics Reports, 433:181-301.

Furlanetto, S. R., Zaldarriaga, M., \& Hernquist, L. (2004b). The Growth of H II Regions During Reionization. ApJ, 613:1-15.

Geil, P. M. \& Wyithe, J. S. B. (2008). The impact of a percolating IGM on redshifted 21-cm observations of quasar HII regions. MNRAS, 386:1683-1694.

Giri, S. K., Mellema, G., Dixon, K. L., \& Iliev, I. T. (2018). Bubble size statistics during reionization from 21-cm tomography. MNRAS, 473:2949-2964.

Gnedin, N. Y. \& Abel, T. (2001). Multi-dimensional cosmological radiative transfer with a Variable Eddington Tensor formalism. New Astronomy, 6:437-455. 
Greig, B. \& Mesinger, A. (2015). 21CMMC: an MCMC analysis tool enabling astrophysical parameter studies of the cosmic $21 \mathrm{~cm}$ signal. MNRAS, 449:4246-4263.

Greig, B. \& Mesinger, A. (2017). The global history of reionization. MNRAS, 465:4838-4852.

Iliev, I. T. et al. (2006). Cosmological radiative transfer codes comparison project - I. The static density field tests. MNRAS, 371:1057-1086.

Iliev, I. T., Mellema, G., Ahn, K., Shapiro, P. R., Mao, Y., \& Pen, U.-L. (2014). Simulating cosmic reionization: how large a volume is large enough? MNRAS, 439:725-743.

Iliev, I. T., Mellema, G., Shapiro, P. R., Pen, U.-L., Mao, Y., Koda, J., \& Ahn, K. (2012). Can 21-cm observations discriminate between high-mass and low-mass galaxies as reionization sources? MNRAS, 423:2222-2253.

Kakiichi, K., Graziani, L., Ciardi, B., Meiksin, A., Compostella, M., Eide, M. B., \& Zaroubi, S. (2017). The concerted impact of galaxies and QSOs on the ionization and thermal state of the intergalactic medium. MNRAS, 468:3718-3736.

Koopmans, L. et al. (2015). The Cosmic Dawn and Epoch of Reionisation with SKA. Advancing Astrophysics with the Square Kilometre Array (AASKA14), page 1.

Lacey, C. \& Cole, S. (1993). Merger rates in hierarchical models of galaxy formation. MNRAS, 262:627-649.

Lewis, A. \& Bridle, S. (2002). Cosmological parameters from CMB and other data: A Monte Carlo approach. PRD, 66(10):103511.

Lewis, A., Challinor, A., \& Lasenby, A. (2000). Efficient Computation of Cosmic Microwave Background Anisotropies in Closed Friedmann-Robertson-Walker Models. ApJ, 538:473476.

Majumdar, S., Pritchard, J. R., Mondal, R., Watkinson, C. A., Bharadwaj, S., \& Mellema, G. (2017). Quantifying the non-Gaussianity in the EoR 21-cm signal through bispectrum. ArXiv e-prints.

Mason, C. A., Treu, T., Dijkstra, M., Mesinger, A., Trenti, M., Pentericci, L., de Barros, S., \& Vanzella, E. (2017). The Universe is Reionizing at $\mathrm{z}^{\sim} 7$ : Bayesian Inference of the IGM Neutral Fraction Using Ly $\alpha$ Emission from Galaxies. ArXiv e-prints:1709.05356.

McQuinn, M., Lidz, A., Zahn, O., Dutta, S., Hernquist, L., \& Zaldarriaga, M. (2007). The morphology of HII regions during reionization. MNRAS, 377:1043-1063.

Mesinger, A. (2010). Was reionization complete by z 5-6? MNRAS, 407:1328-1337.

Mesinger, A., Aykutalp, A., Vanzella, E., Pentericci, L., Ferrara, A., \& Dijkstra, M. (2015). Can the intergalactic medium cause a rapid drop in $\operatorname{Ly} \alpha$ emission at $\mathrm{z}<6$ ? MNRAS, 446:566-577.

Mesinger, A. \& Furlanetto, S. (2007). Efficient Simulations of Early Structure Formation and Reionization. ApJ, 669:663-675.

Mesinger, A., Furlanetto, S., \& Cen, R. (2011). 21CMFAST: a fast, seminumerical simulation of the high-redshift 21-cm signal. MNRAS, 411:955-972.

Mesinger, A., Greig, B., \& Sobacchi, E. (2016). The Evolution Of $21 \mathrm{~cm}$ Structure (EOS): public, large-scale simulations of Cosmic Dawn and reionization. MNRAS, 459:2342-2353.

Mesinger, A., McQuinn, M., \& Spergel, D. N. (2012). The kinetic Sunyaev-Zel'dovich signal from inhomogeneous reionization: a parameter space study. MNRAS, 422:1403-1417.

Mitra, S., Choudhury, T. R., \& Ferrara, A. (2015). Cosmic reionization after Planck. MNRAS, 454:L76-L80.

Mortonson, M. J. \& Hu, W. (2008). Model-Independent Constraints on Reionization from LargeScale Cosmic Microwave Background Polarization. ApJ, 672:737-751.

Mutch, S. J., Geil, P. M., Poole, G. B., Angel, P. W., Duffy, A. R., Mesinger, A., \& Wyithe, J. S. B. (2016). Dark-ages reionization and galaxy formation simulation III: Modelling galaxy formation and the Epoch of Reionization. MNRAS, 462:250-276.

Ocvirk, P. et al. (2016). Cosmic Dawn (CoDa): the First Radiation-Hydrodynamics Simulation of Reionization and Galaxy Formation in the Local Universe. MNRAS, 463:1462-1485.

Pacucci, F., Mesinger, A., Mineo, S., \& Ferrara, A. (2014). The X-ray spectra of the first galaxies: $21 \mathrm{~cm}$ signatures. MNRAS, 443:678-686.

Parsons, A. R. et al. (2010). The Precision Array for Probing the Epoch of Re-ionization: Eight Station Results. AJ, 139:1468-1480.

Partl, A. M., Maselli, A., Ciardi, B., Ferrara, A., \& Müller, V. (2011). Enabling parallel computing in CRASH. MNRAS, 414:428-444. 
Planck Collaboration, . (2016). Planck 2016 intermediate results. XLVII. Planck constraints on reionization history. ArXiv e-prints:1605.0350\%.

Price, L. C., Trac, H., \& Cen, R. (2016). Reconstructing the redshift evolution of escaped ionizing flux from early galaxies with Planck and HST observations. ArXiv e-prints:1605.03970.

Reichardt, C. L. (2016). Observing the Epoch of Reionization with the Cosmic Microwave Background. In Mesinger, A., editor, Astrophysics and Space Science Library, volume 423 of Astrophysics and Space Science Library, page 227.

Santos, M. G., Ferramacho, L., Silva, M. B., Amblard, A., \& Cooray, A. (2010). Fast large volume simulations of the $21-\mathrm{cm}$ signal from the reionization and pre-reionization epochs. MNRAS, 406:2421-2432.

Seljak, U. \& Zaldarriaga, M. (1996). A Line-of-Sight Integration Approach to Cosmic Microwave Background Anisotropies. ApJ, 469:437.

Shimabukuro, H., Yoshiura, S., Takahashi, K., Yokoyama, S., \& Ichiki, K. (2016). $21 \mathrm{~cm}$ line bispectrum as a method to probe cosmic dawn and epoch of reionization. MNRAS, 458:30033011.

Sobacchi, E. \& Mesinger, A. (2014). Inhomogeneous recombinations during cosmic reionization. MNRAS, 440:1662-1673.

Tingay, S. J. et al. (2013). The Murchison Widefield Array: The Square Kilometre Array Precursor at Low Radio Frequencies. PASA, 30:7.

Trac, H. \& Cen, R. (2007). Radiative Transfer Simulations of Cosmic Reionization. I. Methodology and Initial Results. ApJ, 671:1-13.

Trac, H. Y. \& Gnedin, N. Y. (2011). Computer Simulations of Cosmic Reionization. Advanced Science Letters, 4:228-243.

van Haarlem, M. P. et al. (2013). LOFAR: The LOw-Frequency ARray. A $\& A$, 556:A2.

Watkinson, C. A. \& Pritchard, J. R. (2014). Distinguishing models of reionization using future radio observations of 21-cm 1-point statistics. MNRAS, 443:3090-3106.

Xu, H., Wise, J. H., Norman, M. L., Ahn, K., \& O'Shea, B. W. (2016). Galaxy Properties and UV Escape Fractions during the Epoch of Reionization: Results from the Renaissance Simulations. ApJ, 833:84.

Zahn, O., Lidz, A., McQuinn, M., Dutta, S., Hernquist, L., Zaldarriaga, M., \& Furlanetto, S. R. (2007). Simulations and Analytic Calculations of Bubble Growth during Hydrogen Reionization. ApJ, 654:12-26.

Zahn, O., Mesinger, A., McQuinn, M., Trac, H., Cen, R., \& Hernquist, L. E. (2011). Comparison of reionization models: radiative transfer simulations and approximate, seminumeric models. MNRAS, 414:727-738. 